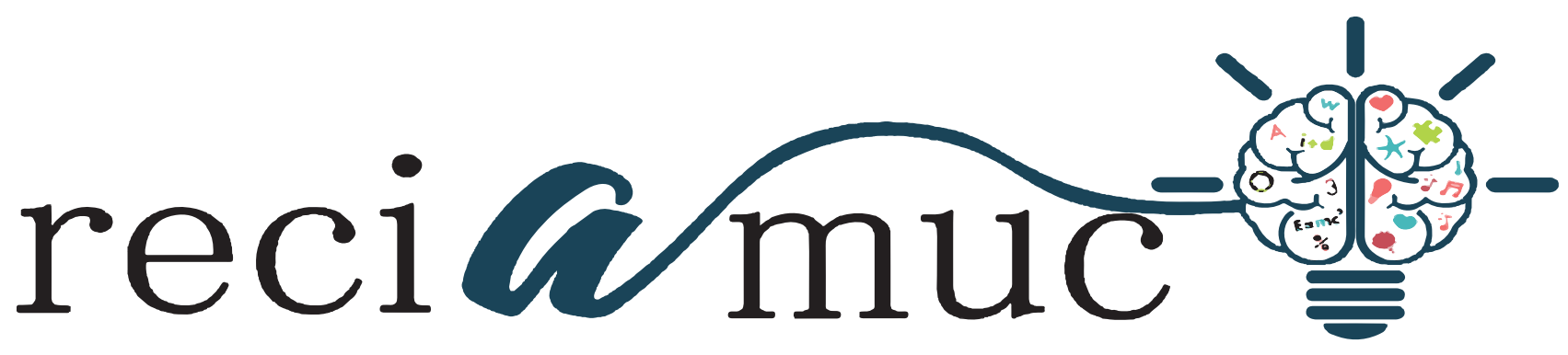

DOI: $10.26820 /$ reciamuc/4.(3).julio.2020.4-16

URL: https://reciamuc.com/index.php/RECIAMUC/article/view/494

EDITORIAL: Saberes del Conocimiento

REVISTA: RECIAMUC

ISSN: 2588-0748

TIPO DE INVESTIGACIÓN: Artículo de Revisión

Código UNESCO: 32 Ciencias Médicas; 3201 Ciencias Clínicas;

3201.04 Patología Clínica

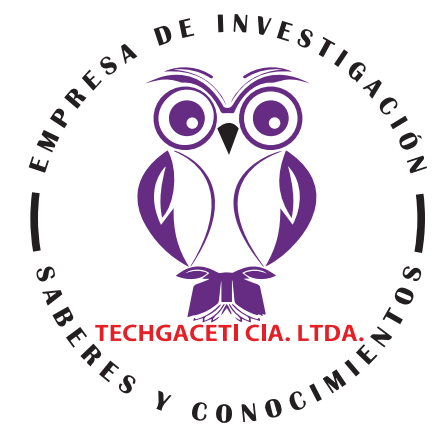

PAGINAS: 4-16

\title{
Lesión de Dieulafoy
}

Dieulafoy's injury

Lesão de Dieulafoy

\section{Verónica Ayling Lama Asinc'; Marjorie Zulay Castro Holguín'; Jonathan Livingston Morante Mendoza33; Amada Alaja García Bermúdes ${ }^{4}$}

RECIBIDO: 18/01/2020 ACEPTADO: 20/03/2020 PUBLICADO: 30/04/2020

1. Médico; Investigador Independiente; Guayaquil, Ecuador; aylinglamaasinc@gmail.com; (D) https://orcid. org/0000-0002-1726-8367

2. Médico; Investigador Independiente; Guayaquil, Ecuador; marjorie88@live.com; iD https://orcid.org/00000003-0852-7491

3. Médico; Investigador Independiente; Guayaquil, Ecuador; jonathan_6688@hotmail.com; iD https://orcid. org/0000-0003-4339-3935

4. Médico; Investigador Independiente; Guayaquil, Ecuador; amaditagb23@gmail.com; iD https://orcid.org/00000002-4117-8206

\section{CORRESPONDENCIA}

Verónica Ayling Lama Asinc

aylinglamaasinc@gmail.com

Guayaquil, Ecuador 


\title{
RESUMEN
}

La Lesión de Dieulafoy consiste en una lesión vascular infrecuente en la cual una arteriola submucosa de recorrido tortuoso y de diámetro diez veces mayor de lo normal finaliza su recorrido cerca de la superficie mucosa. Esta condición, también conocida como: ectasia vascular de la arteria del estómago, exulceratio simplex, malformación arterial (o arteriovenosa) de Dieulafoy, erosión gástrica de Dieulafoy, ulcera de Dieulafoy, enfermedad de Dieulafoy, puede ser responsable de hemorragias digestivas altas en un rango de 2 a 5\%, así como también del frecuente y potencialmente mortal sangrado masivo en los pacientes quirúrgicamente intervenidos, en proporciones que oscilan en 1 y $2 \%$. Por lo general, esta lesión ocurre el $75 \%$ de las veces en el estómago proximal. y en zonas extragástricas el $25 \%$ restante, concentrándose una mayor incidencia en el duodeno, luego en el colon y de último e el recto. El objetivo del presente estudio, consiste fundamentalmente en la exploración de la literatura cientificoacadémica reciente mediante el uso de diversos medios digitales de información, a fines de identificar y referir fuentes útiles para desarrollar aproximadamente lo referente a la lesión de Dieulafoy. Los resultados obtenidos satisfacen el objetivo planteado ya que se pudo constatar la existencia de suficientes fuentes documentales, no obstante, abarcar todo lo concerniente a este tema con este tipo de investigaciones, es materialmente inalcanzable en el corto plazo por lo que la misma se delimitado a una breve aproximación temática. De la revisión es posible concluir, entre otras cosas, que: desde una perspectiva amplia, existe suficiente concordancia entre las distintas acepciones encontradas en la literatura consultada. Esta misma tendencia se mantiene respecto a las causas (etiología) síntomas y signos, y diagnóstico, sólo se notó cierta variación entre las opiniones relativas a la terapéutica.

Palabras clave: Lesión vascular, método hemostático, exulceratio simplex, arteriola abierta, submucosa gástrica.

\begin{abstract}
Dieulafoy's lesion consists of an infrequent vascular lesion in which a tortuous submucosa arteriole with a diameter ten times greater than normal ends its journey near the mucosal surface. This condition, also known as: vascular ectasia of the artery of the stomach, exulceratio simplex, arterial (or arteriovenous) malformation of Dieulafoy, gastric erosion of Dieulafoy, Dieulafoy's ulcer, Dieulafoy's disease, may be responsible for upper digestive bleeding in a range from 2 to $5 \%$, as well as the frequent and life-threatening massive bleeding in surgically operated patients, in proportions ranging from 1 to $2 \%$. This injury usually occurs $75 \%$ of the time in the proximal stomach. and in extragastric areas the remaining $25 \%$, concentrating a higher incidence in the duodenum, then in the colon and lastly in the rectum. The objective of the present study, consists fundamentally in the exploration of the recent scientific-academic literature through the use of various digital information media, in order to identify and refer useful sources to develop approximately what refers to the injury of Dieulafoy. The results obtained satisfy the stated objective since it was possible to verify the existence of sufficient documentary sources, however, covering everything related to this topic with this type of research, is materially unattainable in the short term, for which reason it was limited to a brief thematic approach. From the review it is possible to conclude, among other things, that: from a broad perspective, there is sufficient agreement between the different meanings found in the literature consulted. This same trend is maintained regarding the causes (etiology) symptoms and signs, and diagnosis, only some variation was noted between the opinions regarding therapeutics.
\end{abstract}

Keywords: Vascular injury, hemostatic method, simplex exulceration, open arteriole, gastric submucosa.

\section{RESUMO}

A lesão de Dieulafoy consiste em uma lesão vascular pouco frequente, na qual uma arteríola submucosa tortuosa com diâmetro dez vezes maior que o normal termina sua jornada perto da superfície da mucosa. Essa condição, também conhecida como: ectasia vascular da artéria do estômago, exulceratio simplex, malformação arterial (ou arteriovenosa) de Dieulafoy, erosão gástrica de Dieulafoy, úlcera de Dieulafoy, úlcera de Dieulafoy, doença de Dieulafoy, pode ser responsável pelo sangramento digestivo superior em uma faixa de 2 a 5\%, bem como o sangramento maciço frequente e com risco de vida em pacientes operados cirurgicamente, em proporções que variam de $1 \mathrm{a} 2 \%$. Essa lesão geralmente ocorre $75 \%$ das vezes no estômago proximal. e nas áreas extragástricas os 25\% restantes, concentrando maior incidência no duodeno, depois no cólon e, por último, no reto. O objetivo do presente estudo consiste fundamentalmente na exploração da literatura científico-acadêmica recente, por meio do uso de diversos meios de informação digital, a fim de identificar e encaminhar fontes úteis para desenvolver aproximadamente o que se refere à lesão de Dieulafoy. Os resultados obtidos atendem ao objetivo declarado, uma vez que foi possível verificar a existência de fontes documentais suficientes, porém, cobrindo tudo relacionado a esse tópico com esse tipo de pesquisa, é materialmente inatingível a curto prazo, motivo pelo qual se limitou a breve abordagem temática. A partir da revisão, é possível concluir, entre outras coisas, que: de uma perspectiva ampla, há concordância suficiente entre os diferentes significados encontrados na literatura consultada. Essa mesma tendência é mantida em relação aos sintomas e sinais de causas (etiologia) e diagnóstico, apenas algumas variações foram observadas entre as opiniões sobre a terapêutica.

Palavras-chave: Lesão vascular, método hemostático, exulceração simplex, arteríola aberta, submucosa gástrica. 


\section{Introducción}

Los vasos sanguíneos anómalos que se hallan en la capa mucosa o submucosa del aparato digestivo son característicos de algunos síndromes congénitos o adquiridos y, aunque estos pueden causar una periódica hemorragia, en muy pocos casos llega a ser masiva (Ansari, 2018). Uno de esos síndromes ha sido identificado como: Lesión de Dieulafoy (LD).

Es sabido que el origen de esta condición sobrepasa ya los 135 años de haber sido descubierta por primera vez por Gallard en la década de los años 80 del siglo antes pasado cuando le denominó: ectasia vascular de la arteria del estómago. Luego, fue el patólogo parisino Paul Dieulafoy quien catorce años después pudo definir más detalladamente esta complicación algo extraña, al identificar mediante una autopsia de uno de sus pacientes, una ulceración superficial que se trataba de una arteriola abierta ubicada dentro de la submucosa gástrica, concluyendo en base a ello entonces que, el daño no era típicamente consistente con el de una úlcera gástrica por lo cual le denominó "exulceratio simplex", y que más tarde termina conociéndose como antes se ha referido. (Khan, Mahmad, Gobrial, Onwochei, \& Shah, 2015, pág. 201)

A lo largo de los años ha recibido muchos nombres y fue hasta 1966 que apareció designada como Dieulafoy en la literatura inglesa, difundiéndose y aceptándose con el nombre de malformación arterial de Dieulafoy, o como malformación arteriovenosa de Dieulafoy, erosión gástrica de Dieulafoy, ulcera de Dieulafoy, enfermedad de Dieulafoy y lesión de Dieulafoy. (Durán \& Girón, 2019, pág. 15)

De conformidad al manual Medicina Interna Basada en la Evidencia 2019/20 la LD consiste en:

una lesión vascular infrecuente (causa entre el 1-2 \% de los casos de hemorragia digestiva alta aguda), en la cual una arteriola submucosa de recorrido tortuoso y de diámetro diez veces mayor de lo normal (1-3 $\mathrm{mm}$ ) finaliza su recorrido cerca de la superficie mucosa. Se cree que puede tener un carácter congénito.

Aunque el criterio de Massinha, Cunha, \& Tomé (2020) concuerda con lo dicho respecto a la muy baja incidencia de esta causa en las hemorragias gastrointestinales (HGl) en general y en el sangrado no varicoso en el tracto gastrointestinal superior, no deja de advertir su riesgo pues la tasa de mortalidad por DL es muy parecida cuando se compara a las de otras causas de HGI. Adicionalmente indicaron que:

Antes de la era de la endoscopia, la mortalidad por sangrado debido a LD era alta, ya que el único tratamiento existente era la ligadura quirúrgica del vaso aberrante o la gastrectomía subtotal (o total). Por lo tanto, el progreso de las técnicas endoscópicas hemostáticas ofreció un enfoque terapéutico diferente y efectivo, con una reducción en la tasa de mortalidad de alrededor del 10\%. (pág. 238)

En este mismo sentido, en 2015, Khan et al. referían que la endoscopia continuaba implementándose fundamentalmente como enfoque diagnóstico principal en LD y de casi todas las formas de HGl. Aunado a ello, también destacaron el efecto trascendente de dicha metodología en el ámbito terapéutico de LD mediante el uso de bandas laparoscópicas, inyecciones con epinefrina y adrenalina, y termocoagulación; no obstante, reconocieron las limitaciones de ambos aspectos en cuanto a las estadísticas exitosas. (Khan, Mahmad, Gobrial, Onwochei, \& Shah, 2015, pág. 201)

Por lo general, el tratamiento endoscópico de la LD consiste en técnicas de: inyección de sustancias especiales, entre estas, la adrenalina diluida y polidocanol, y excepcionalmente, cianoacrilato; termocoagulación, tratándose de, coagulación con argón plasma, electrocoagulación, sonda térmica; mecánicas, tales como, clips hemostáticos, 
bandas hemostáticas o la combinación de varias de estas técnicas, con las que en más de un 70 y menos del $95 \%$ de los casos se alcanzan los resultados más óptimos (Medicina Interna Basada en la Evidencia 2019/20)

El objetivo del presente estudio, consiste fundamentalmente en la exploración de la literatura cientificoacadémica reciente mediante el uso de diversos medios digitales de información, a fines de identificar y referir fuentes útiles para desarrollar aproximadamente lo referente a la lesión de Dieulafoy, por lo que como resultado se procurará obtener referir algunas de sus acepciones, posibles causas, signos y síntomas, forma de diagnosticarla y tratamiento.

\section{Materiales y Métodos}

Esta investigación se ha delimitado a la búsqueda y revisión de literatura científica y académica vigente, la cual se encuentre accesible completamente a través del uso de las siguientes bases de datos: NCBI, PubMed, SciELO, Medigraphic, Elsevier, Dialnet, Karger y otras. Igualmente fueron consultados otros repositorios de sitios web de alcance nacional, regional e internacional, entre estos los de: revistas científicas, universidades, organismos, sociedades o asociaciones profesionales, y demás entes de trascendencia en el ámbito de la salud y medicina humana.

Los criterios de búsqueda utilizados respondieron fundamentalmente a las expresiones "lesión dieulafoy" y "exulceratio simplex", con los cuales se fueron obteniendo distintos tipos de material bibliográfico. Se consideró prudente solamente revisar el contenido que estuviera caracterizado como artículo científico; tesis de grado, posgrado y doctorado; e-books; guías y ensayos clínicos; consensos y protocolos; noticias, boletines, folletos e informes científicos; planes, proyectos y cualquier otra clase de documento que se tratase de algún tipo de investigación, a fines de identificar, seleccionar y referir exclusivamente aquellas fuentes que sirviesen para exponer sobre el tema en cuestión.

Adicionalmente se decidió aplicar otros criterios de selección, tales como: fecha de publicación en los últimos 10 años (incluyendo el corriente), idioma español e inglés, área de especialidad en medicina humana y ciencias de la salud, y especificidad o correlación temática.

Los recursos bibliográficos que fueron excluidos correspondieron a editoriales, cartas al editor y otros tipos de materiales menos estructurados o informales, debido a que, per se, poseían muy bajo nivel de evidencia necesaria para este tipo de investigaciones.

Cabe señalar que toda la evidencia definitivamente escogida distribuida y compartida entre los miembros del equipo investigador, a fines de que, consecuentemente, fuera sometida a una lectura crítica y análisis interpretativo correspondiente; tanto de manera individual como grupal, para entonces así alcanzar la concreción de las ideas que han quedado plasmadas en las otras secciones de la presente entrega, y que, por cierto, en todo momento se fueron logradas de manera consensuada.

\section{Resultados}

Una lesión de Dieulafoy, también denominada "arteria persistente de calibre", es una causa relativamente rara, pero potencialmente mortal, de hemorragia del tracto gastrointestinal. Es difícil determinar con precisión su verdadera incidencia en la población general, ya que permanecen en silencio hasta la presentación y, aun así, puede suponer un desafío diagnóstico. Se cree que representan sólo el 1-2\% de la hemorragia Gl aguda, pero son, posiblemente, poco reconocido[sic] en lugar de ser verdaderamente raras. La falta de conciencia sobre la lesión de Dieulafoy contribuye a su "rareza", aumento de la morbilidad y también a la mortalidad previamente reportada de hasta un $80 \%$ asociada con esta lesión. Raras o no, la naturaleza precaria de la pre- 
sentación hace que sea necesario incluirlas en el diagnóstico diferencial de cualquier hemorragia gastrointestinal aguda. (Baxter \& Aly, 2010)

Para Botello et al. (2013), la LD consiste en un vaso arterial histológicamente normal, de gran tamaño, que de manera anómala mantiene su calibre (1-3 mm) a lo largo de un recorrido sinuoso desde la submucosa hasta la mucosa, donde penetra por un defecto mínimo.

Continuó indicando que esta condición puede ser responsable de hemorragias digestivas altas en un rango de 2 a 5\%, así como también del frecuente y potencialmente mortal sangrado masivo en los pacientes quirúrgicamente intervenidos, en proporciones que oscilan en 1 y $2 \%$. Adicionalmente aportó que este tipo de lesiones ocurren el $75 \%$ de las veces en el estómago proximal y en zonas extragástricas el 25\% restante, siendo su mayor incidencia en el duodeno, luego en el colon y de último las rectales. (Botello et al.; 2013). En Soria et al. (2018) también se afirma que "Las LD se encuentran principalmente en estómago (80\%), aunque se han reportado en lugares como esófago, duodeno (15\%), colon (2\%) y recto (menos del 2\%)" (pág. 374)

Con mayor frecuencia causa hemorragia gastrointestinal superior, pero también puede causar hemorragia gastrointestinal media (definida como hemorragia localizada entre la ampolla de Vater y el ciego), y rara vez causa hemorragia gastrointestinal inferior, dependiendo de la ubicación de la lesión. (Nojkov \& Cappell, 2015)

Rojas, Carvajal, Prieto, \& Aponte (2016) particularmente han destacado que la LD es una de las causas de hemorragia digestiva alta oculta, que puede ser potencialmente mortal y representa el $0,1 \%$ de las hemorragias agudas. La mayoría de LD se encuentra en el estómago, dentro de los $6 \mathrm{~cm}$ de la unión gastroesofágica; su localización extragástrica es rara (...)
La falta de conocimiento acerca de la lesión de Dieulafoy aumenta la morbilidad y mortalidad de los pacientes que la padecen. (págs. 292-293)

"La LD es una arteria de un calibre anormalmente grande que penetra en la pared intestinal y, en ocasiones, erosiona la mucosa y provoca hemorragia masiva. Su localización principal es en el estómago." (Ansari, 2018)

Las lesiones de Dieulafoy (LD) se definen como una anomalía vascular solitaria con erosión de la mucosa que produce un sangrado proveniente de una arteria submucosa anormalmente larga y tortuosa, esta arteria no se estrecha a medida que se extiende hacia la mucosa por lo que el sangrado generalmente es severo. (Soriaet al.; 2018; pág. 374)

Massinha et al. (2020) explican que la LD se trata de un brote de la "arteria inusualmente grande y tortuosa" (pág. 238) que se extiende a lo largo de la muscularis mucosa del tracto gastrointestinal, y que mayormente se presenta en el estómago proximal. Este vaso sobresaliente puede entrar en erupción en la luz como una imperfección menor de la mucosa, aunque los mecanismos patogénicos aún no se entienden completamente (Ver Figura 1) 


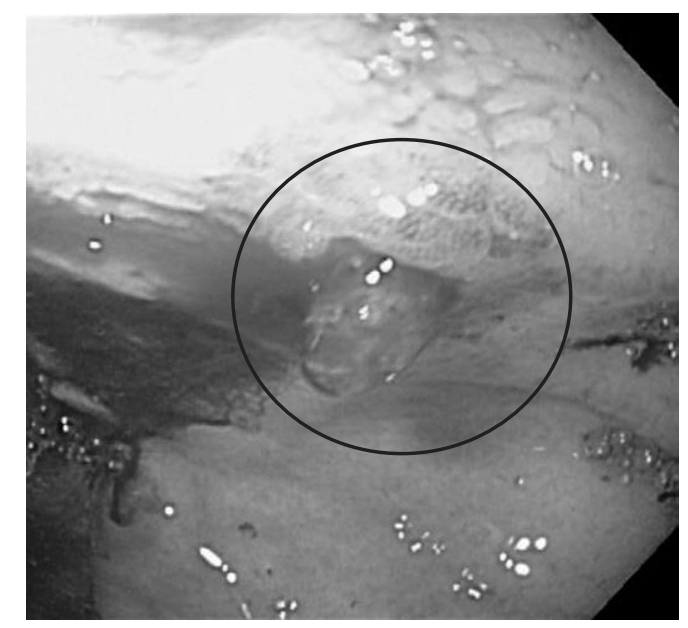

Figura 1. Lesión de Dieulafoy gástrica en la pared anterior del cuerpo proximal

Fuente: Botello et al. (2013)

\section{Causas}

En su investigación Botello et al. (2013) fundamenta que todavía es un misterio la patogénesis de esta lesión pues hasta ese momento los estudios no eran concluyentes respecto a la asociación entre ésta y el consumo de alcohol o antiinflamatorios no esteroides, o inclusive con la enfermedad ulcerosa péptica, pero, lo que sí estaba claro era la conjunción entre las lesiones vasculares en el aparato digestivo y las patologías sistémicas (tales como la cirrosis hepática y la insuficiencia renal crónica), como también con las angiodisplasias o la ectasia vascular antral. La razón de ello, contnuaron explicando, quizá se debía a que este tipo de trastornos afecten la angiogénesis normal propiciando el surgimiento de neovasos arteriales aberrantes de calibre persistente que, en condiciones de estrés de la mucosa digestiva, es posible que se deterioren $y$ induzcan la hemorragia. Adicionalmente sustentaron que:

Varios estudios han puesto de manifiesto otro tipo de comorbilidades asociadas, como: enfermedad de von Willebrand, padecimientos cardiacos (estenosis aórtica, enfermedad vascular y cardiomiopatía isquémica congestiva), enfermedades respiratorias, poliposis intestinal, colestasis intrahepática, trastornos autoinmunitarios, páncreas ectópico, diabetes mellitus tipo 2 , tirotoxicosis y atrofia gástrica. También se ha querido explicar la lesión de Dieulafoy a través de la teoría de Scott-Boyle (obstrucción intermitente del drenaje venoso).

[...] Algunos autores suponen que la presión focal a partir de estos vasos de calibre persistente adelgaza la mucosa suprayacente y por isquemia produce erosión de la pared vascular y la hemorragia subsecuente. (Botello, y otros, 2013, pág. 325)

En definitiva, éstos expertos aseguran que esa lesión puede que no derive en nada identificable en el transcurso de un considerable tiempo y de repente expedir el sangrado en razón de las siguientes posibilidades: los traumatismos de la mucosa gástrica; prolapso de la mucosa del antro gástrico hacia el píloro; contracciones intensas frecuentes; gastritis reactiva y otros mecanismos que dañan la pared del vaso. De la misma manera Ledo Rodríguez \& Ledo Barro (2012) especifican que, respecto a la arteria, no se sabe exactamente que ocasiona su rotura, sin embargo, refieren que en teoría se manejan las ideas de que es porque se produce una mínima erosión de la mucosa que la recubre, y ésta a su vez podría deberse a: una atrofia (estrecha- 
miento) de la mucosa, a la isquemia (falta de flujo sanguíneo) de ésta, o por trombosis de la arteria (obstrucción por un coágulo).

En el estudio de Khan et al. (2015) adicionalmente se afirma que no se había determinado hasta ese momento una verdadera etiología de la ruptura de la mucosa. Ciertamente se llegó a pensar que se relacionaba con un aneurisma heredado, pero tras estudios posteriores no se ha podido evidenciar "un vínculo con los aneurismas, la arteriosclerosis, la disfunción del tejido elástico o la vasculitis." (pág. 202)

\section{Síntomas (Signos)}

En 2012 Ledo Rodríguez \& Ledo Barro expusieron que la LD se presenta como una hemorragia a causa del rompimiento de la arteria antes mencionada. Cuando ésta surge a nivel del tubo digestivo, el síntoma se puede registrar mediante un vómito con sangre o aspecto de «posos de café» (hematemesis), evacuaciones de color negro (melenas), de negro con el fondo rojizo (hematoquecia) o deposiciones con pura sangre (o fresca) conocida como rectorragias. Comúnmente, esta pérdida de sangre ocurre de forma aguda y naturalmente ceda, sin embargo, es posible que reincida, e inclusive, de forma masiva.

El cuadro clínico se distingue por una hemorragia digestiva que puede llevar a pérdidas de volumen que van desde la clase II hasta la clase IV del Colegio Americano de Cirujanos; otra forma de presentación es el inicio súbito o con episodios intermitentes e indoloros de sangrado.

Por lo general, los pacientes tienen antecedente de dispepsia o enfermedad ulcerosa péptica. Las presentaciones clásicas incluyen hematemesis sola (28\%), hematemesis con melena (51\%) y melena sola (18\%). El sangrado de la lesión de Dieulafoy con frecuencia es de alivio espontáneo, pero el sangrado arterial que pone en peligro la vida ocurre en $10 \%$ de los casos, aproximadamente. (Botello, y otros, 2013, págs. 325-
326)

\section{Diagnóstico}

Khan et al. (2015) resaltan el dilema que surge tras el diagnóstico de la LD, y esto es debido a que, por una parte, esta lesión puede surgir en multiples sitios, en algunos casos, incluso inaxesibles mediante endoscopia (como el yeyuno o el íleon). y por la otra, aunado a que es muy común que esta misma consista en un sangramiento intermitente, es lo diculta oportuno y preciso diagnóstico. Todo este habitual escenario coincide también con la posibilidad de que tales lesiones pueden ser tan pequeñas y relativamente discretas que llegan a pasar sedapercibidas aún después de varias sesiones endoscópicas y otras medidas de diagnóstico; entonces he allí la disyuntiva que posiblemente genere el incremento de la mortalidad de estos pacientes en comparación con los de otros tipos de sangrado gastrointestinal que pueden manejarse fácilmente. En detalle explican que:

La prueba diagnóstica más utilizada es una esofagogastroduodenoscopia (EGD), que demostraría una protuberancia pigmentada del muñón de un vaso que puede estar rodeada de erosión mínima y falta de ulceración. Esta protuberancia pigmentada puede tener un color variable, tiene aproximadamente 10-15 mm de ancho, 5-10 mm de altura y solo $50-60 \%$ puede estar sangrando activamente con un chorro o exudación mínima de sangre de la mucosa gastrointestinal. Varios pacientes pueden requerir endoscopias repetitivas, ya que las medidas de diagnóstico iniciales pueden no revelar una fuente; sin embargo, la hemoglobina del paciente puede continuar disminuyendo. Es posible que no se detecte porque un coágulo adherente puede ocluirlo y, a veces, la única forma de exponerlo directamente es lavar el coágulo con una perfusión endoscópica moderada, aunque esto no es muy recomendable.

Se ha descubierto que la EGD inicial es diagnóstica en solo alrededor del $70 \%$ de 
los casos, ya que las lesiones son relativamente pequeñas e intermitentemente activas. También pueden ubicarse entre pliegues, cubrirse con un coágulo, ubicarse debajo del contenido gástrico u ocultarse debido a charcos de sangrado masivo. Los criterios endoscópicos que a veces se han utilizado para definir DL incluyen los siguientes: 1) chorro arterial activo o flujo micropulsátil de un defecto $<3 \mathrm{~mm}$ en la mucosa o la mucosa normal circundante; 2) visualización del vaso sobresaliente con o sin sangrado activo dentro del pequeño defecto de la mucosa o a través de la mucosa circundante normal; y 3) aspecto de coágulo fresco y densamente adherente con un punto de unión de mucosa diminuto. Aunque el rendimiento final del diagnóstico de EGD es del $70 \%$ para DL, los hallazgos diagnósticos iniciales son mucho menores. Aproximadamente el $49 \%$ de las lesiones se identifican durante la EGD inicial y el 33\% puede requerir más de una EGD para localizar correctamente la fuente activa de sangrado.

Como EGD tiene sus limitaciones, también se ha utilizado la enteroscopia de empuje que puede tener un mayor rendimiento en la identificación de ubicaciones oscuras con DL. La enteroscopia a menudo está indicada después de que la EGD y la colonoscopia no hayan sido diagnósticas. Permite ver el sistema gastrointestinal unos $150 \mathrm{~cm}$ más allá del píloro y es capaz de identificar lesiones duodenales distales o yeyunales proximales. La endoscopia con cápsula inalámbrica también se ha implementado y, aunque se ha considerado mínimamente invasiva, carece del beneficio de la intervención terapéutica. La cápsula endoscópica es una forma de investigar áreas del tracto gastrointestinal que pueden ser difíciles de identificar con EGD e incluso colonoscopia. Con su uso, sigue habiendo limitaciones, ya que la cámara puede perder lesiones más pequeñas, ya que puede no tener la dirección correcta, ya que evita la lesión.

Cuando EGD no pudo localizar la fuente de sangrado, se implementó la angiografía. En la angiografía, la extravasación de contraste en la arteria erosionada puede ser indicativa de la lesión. Es más útil particularmente en lesiones colorrectales donde puede haber una preparación intestinal deficiente, lo que puede oscurecer los resultados de la colonoscopia. No se ha encontrado que un patrón angiográfico sea específico para DL pero puede incluir hallazgos como la visualización de una arteria ectatica no cónica en el sitio de sangrado. La extravasación como se indicó puede ser el único hallazgo a veces. En un estudio, se descubrió que la angiografía era diagnóstica en 11 de 14 pacientes que tenían DL y que se habían sometido a pruebas endoscópicas no diagnósticas.

Otras utilidades de diagnóstico que se han utilizado incluyen el uso adicional de ultrasonido endoscópico. Las características endosonográficas pueden demostrar una gran arteria submucosa, que puede ubicarse en la curva gástrica menor cerca de la unión gastroesofágica y se utiliza principalmente para confirmar la hemostasia endoscópica de una lesión hemorrágica al ilustrar el flujo sanguíneo ausente después de la terapia. Este tipo de práctica no se ha utilizado y a menudo no se recomienda debido a su mayor costo y falta de eficacia.

Una medida de investigación final que se puede utilizar cuando otras técnicas de diagnóstico han fallado y el paciente está clínicamente estable es un examen de glóbulos rojos con tecnecio-99m. La ventaja de lo que conlleva es que el umbral para detectar cualquier extravasación en el intestino es solo el 20\% del requerido por la angiografía. Sin embargo, una vez más, esta modalidad de prueba no se usa con frecuencia debido a la falta de datos que respalden su tasa de éxito en comparación con EGD. (Khan, Mahmad, Gobrial, Onwochei, \& Shah, 2015)

Como complemento, en otro estudio se menciona que, conforme a sus fuentes:

El riesgo de resangrado después del tra- 
tamiento endoscópico puede ser elevado, disminuyendo si se utiliza terapia combinada, Ej. Adrenalina con bandas elásticas o hemoclips. También se describe la utilización de Adrenalina previo a la colocación de endoclips, en los sangrados masivos, para facilitar la visualización del vaso.

Si no se logra controlar la hemorragia, se debe recurrir a cirugía convencional o laparoscópica. (Salazar et al.; 2017; pág. 47)

\section{Tratamiento}

La endoscopia digestiva permite además aplicar diferentes modalidades de tratamiento para lograr frenar el sangrado en la lesión de Dieulafoy. Se considera la técnica de elección para intentar parar la hemorragia, siendo efectiva en el $90 \%$ de los casos. Para conseguirlo, se pueden emplear métodos térmicos (empleando corriente eléctrica o gas argón para coagular el vaso sangrante), de inyección (utilizando sustancias como la adrenalina) o mecánicos (con la utilización de bandas elásticas o clips). Ninguno de estos es claramente superior al otro, por lo que su empleo dependerá de la disponibilidad y de la experiencia o preferencia del endoscopista. Se recomienda la combinación de dos de estos métodos. El riesgo de que vuelva a aparecer la hemorragia después del tratamiento endoscópico puede ser elevado y varía entre un 9 y un $40 \%$. En estos casos, es posible volver a utilizar la endoscopia para intentar controlarlo o bien, utilizar otra opción de tratamiento. Dentro de estas alternativas que se utilizan cuando la endoscopia fracasa se encuentra la angiografía. Mediante esta técnica es posible embolizar aquellos que están sangrando (impedir que fluya la sangre a su través).

Cuando esta opción terapéutica no logra controlar la hemorragia, se debe recurrir a la cirugía convencional o laparoscópica. En el caso de que se opte por la laparoscopia, la endoscopia digestiva podrá ayudar a localizarla lesión (con la colocación de clips o la inyección de sustancias colorantes), lo que facilitará el tratamiento quirúrgico. Este, habitualmente, consiste en la sutura del vaso sangrante o la resección de la zona donde se sitúa esta lesión. (Ledo Rodríguez \& Ledo Barro, 2012)

Las opciones de tratamiento dependen de una variedad de factores que incluyen la presentación del paciente, el sitio de la lesión y las técnicas de diagnóstico utilizadas. Los métodos endoscópicos siguen siendo el tratamiento de elección utilizado con mayor frecuencia en las lesiones de fácil acceso. Con el uso de medidas endoscópicas, las opciones de tratamiento se dividen en tres grupos: 1) electrocoagulación térmica, coagulación con sonda de calor y coagulación con plasma de argón; 2) inyección regional-inyección local de epinefrina y escleroterapia; y 3) bandas mecánicas y hemoclip.

Antes de implementar estas técnicas curativas, se debe hacer un intento para que el paciente sea hemodinámicamente estable. Es vital concentrarse en la reanimación de volumen para evitar las consecuencias del daño del órgano terminal. Se insertan múltiples líneas intravenosas de gran diámetro y se realiza una reanimación de volumen con solución cristalina, ya sea con solución salina normal o timbres de lactato. Dependiendo del nivel de anemia del paciente, a menudo se requiere la transfusión de glóbulos rojos empaquetados y en la mayoría de los pacientes con DL se ha demostrado que requiere tres o incluso más unidades. $\mathrm{DL}$ requiere terapia hemostática, ya que a menudo puede ocurrir un nuevo sangrado y el diagnóstico, específicamente la fuente del sangrado, puede no ser descubierto inicialmente.

Como se indicó, la endoscopia terapéutica es el tratamiento primario y puede lograr la hemostasia inicial en aproximadamente el 90\% de las lesiones que son accesibles y puede disminuir las tasas de reangrado a menos del $10 \%$ en los primeros 7 días. Con el uso de procedimientos hemostáticos en- 
doscópicos, se discutirán las diversas técnicas de tratamiento.

La inyección de epinefrina y la escleroterapia son uno de los tratamientos utilizados para detener el sangrado gastrointestinal. La epinefrina con inyección repetida puede provocar el cese del sangrado. Es una técnica de tratamiento relativamente barata; sin embargo, se ha observado que solo en el tratamiento de DL no se recomienda debido al riesgo de reangrado. La epinefrina alrededor de la lesión se puede usar para reducir el sangrado excesivo, pero también se debe manejar con escleroterapia para las personas con DL. Se ha demostrado que la escleroterapia con etanol o polidocanol controla con éxito el sangrado cuando se usa en cuatro sitios alrededor del vaso y luego dentro del vaso.

La coagulación térmica, otra medida útil, se puede agrupar dependiendo de si se trata de contacto con electrocoagulación bipolar y coagulación con sonda calefactora o sin contacto, entregando corriente monopolar de alta frecuencia a través de un gas conductor a la submucosa. El no contacto se considera ventajoso ya que puede reducir el riesgo de perforación al disminuir la profundidad del daño tisular y debido a la facilidad de su uso. Sin embargo, los métodos térmicos de contacto han sido criticados por la coagulación inadecuada de la lesión cuando está cubierta por sangre, lo que resulta en futuros episodios de sangrado.

La ligadura con banda endoscópica y los hemoclips son las terapias mecánicas más utilizadas. Se ha demostrado que a veces tienen más éxito en comparación con el tratamiento con inyección en el tratamiento de DL. Aunque la tasa de éxito puede ser mayor, solo se aplica a los que se aplican correctamente. Debe reservarse para endoscopistas experimentados, especialmente cuando el ángulo es difícil, ya que los hemoclips desplegados incorrectamente pueden evitar la colocación correcta de futuros clips.
La angiografía con embolización con espuma de gel es una forma relativamente rara de tratamiento, pero puede ser un recurso final efectivo si el tratamiento endoscópico falla. Desafortunadamente, puede haber un riesgo de isquemia en el área irrigada por la arteria embolizada. Por lo tanto, cuando la lesión sangrante es suministrada por múltiples vasos colaterales, esta técnica no es útil. La cirugía es la última opción para pacientes con tratamiento no controlado, pero conlleva una mayor tasa de mortalidad, ya que a menudo estos pacientes pueden ser hemodinámicamente inestables en el momento en que se considera la cirugía. (Khan, Mahmad, Gobrial, Onwochei, \& Shah, 2015, págs. 203-204)

En este mismo sentido, se considera que bien vale la pena referir el estudio de Nojkov \& Cappell (2015) ya que según sus criterios:

Actualmente, la terapia endoscópica ideal para el sangrado reciente de la lesión de Dieulafoy es incierta. Se necesitan ensayos clínicos grandes, prospectivos, comparativos, de diferentes modalidades endoscópicas, pero son difíciles de realizar y completar debido a la rareza relativa de esta lesión. Es razonable, por lo tanto, que los gastroenterólogos adopten técnicas particulares basadas en la experiencia personal y local y las tecnologías disponibles dentro de su suite de endoscopia. El uso de un aerosol para detener el sangrado es una tecnología emocionante debido a la facilidad de uso, pero es experimental y no está probada.

Es probable que la angiografía terapéutica se convierta en una alternativa más viable a la terapia endoscópica, con mayor experiencia con esta tecnología para esta indicación, pero es probable que siga siendo una segunda opción después de la terapia endoscópica fallida debido a la fácil disponibilidad de endoscopia terapéutica en la misma sesión cuando realizando la endoscopia diagnóstica inicial y la muy alta tasa de éxito de la endoscopia terapéutica. Se espera que la terapia endoscópica evolu- 
cione con técnicas aún mejores para la ablación de la lesión o la oclusión mecánica de las lesiones vasculares, como el desarrollo de dispositivos de micro sutura endoscópica clínicamente aplicables.

Aunque la ecografía endoscópica puede ser potencialmente muy útil para identificar si un vaso en una lesión de Dieulafoy tiene flujo activo a través de ella, la adopción generalizada de esta técnica espera reducir el costo de esta tecnología, una mayor disponibilidad de endosonógrafos y la demostración de sus beneficios clínicos a través de ensayos clínicos. La angiografía por tomografía computarizada puede asumir una función diagnóstica mayor después de una endoscopia no diagnóstica frente a un sangrado severo y activo, pero es probable que su función siga siendo limitada debido a la falta de capacidades terapéuticas.

Actualmente, la enteroscopia de globo único y globo doble generalmente se limita a los hospitales terciarios, pero debería estar más disponible en el futuro con la reducción de los costos. Esto puede ofrecer una nueva tecnología para diagnosticar y tratar las lesiones de Dieulafoy del intestino delgado que de otro modo serían difíciles de alcanzar y tratar. La endoscopia con cápsula puede ser más útil para diagnosticar lesiones yeyunoileales con el desarrollo de cápsulas con propulsión activa, mejor resolución de la cámara y baterías más duraderas y más potentes, pero su papel probablemente seguirá siendo limitado para el sangrado de las lesiones yeyunoileales de Dieulafoy debido a la falta de tratamiento capacidades. (pág. 304)

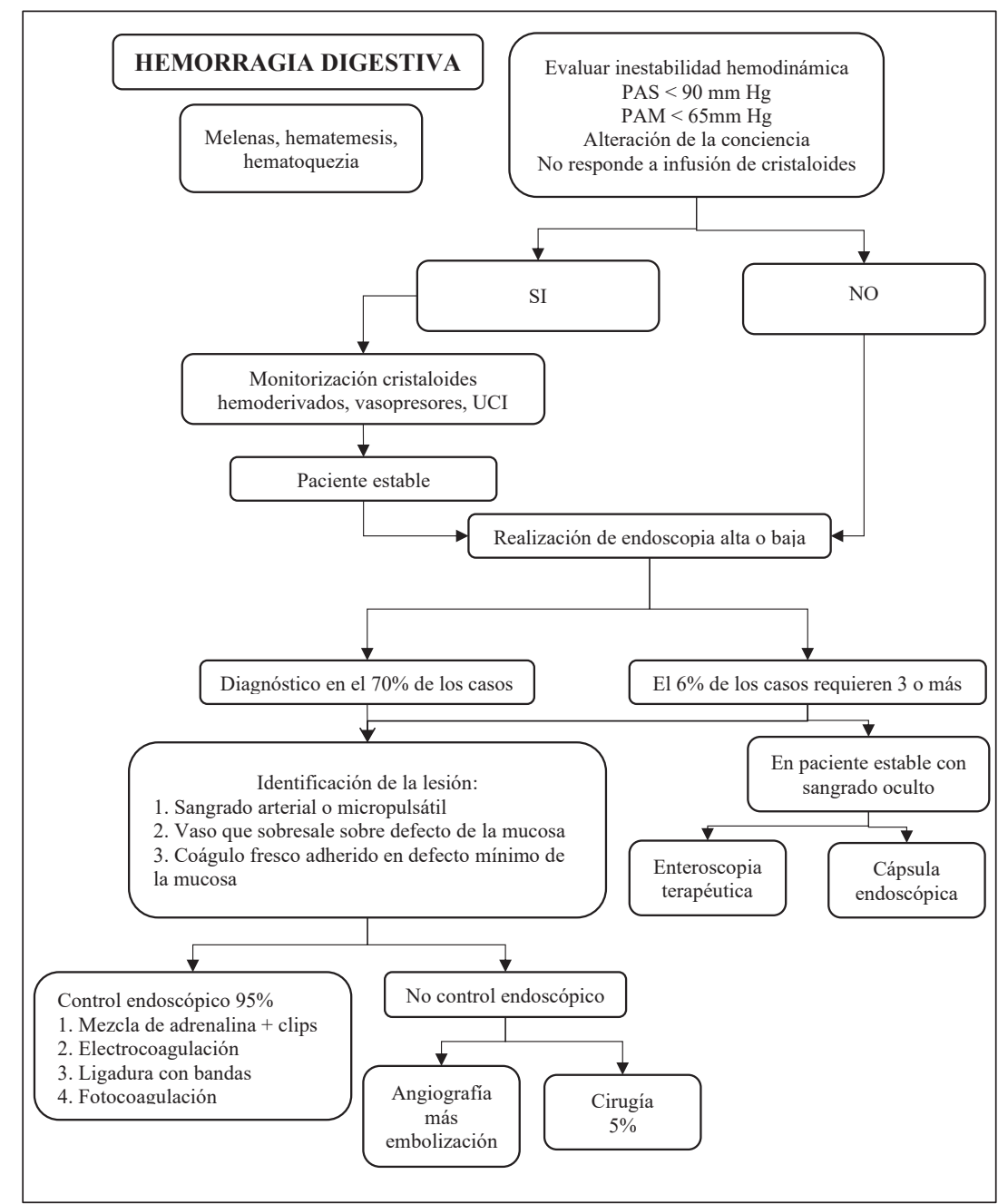

Figura 2. Algoritmo de tratamiento de la lesión de Dieulafoy.

Fuente: Lesión de Dieulafoy de duodeno: hallazgo inusual. Rojas, A. et al. (2016). Revista Colombiana de Gastroenterología. (31)3. Pág. 295. Recuperado de: http:// www.scielo.org.co/pdf/rcg/ v31n3/v31n3a14.pdf 


\section{Conclusión}

De toda la revisión es posible deducir que, desde una perspectiva amplia, existe suficiente concordancia entre las distintas acepciones encontradas en la literatura consultada. Esta misma tendencia se mantiene respecto a las causas (etiología) síntomas y signos, y diagnóstico, sólo se notó cierta variación entre las opiniones relativas a la terapéutica.

De lo anterior, es inevitable atreverse a destacar que, por ejemplo:

- La LD es considerada una condición de rara y aún desconocida etiología, que puede llegar a ser potencialmente mortal. Es la causa de hemorragias gastrointestinales a nivel superior, medio e inferior, reportando una mayor incidencia en el primero de dichos niveles.

- Su diagnóstico puede llegar a ser un verdadero desafío, dado sus imperceptibles síntomas, sus sutiles manifestaciones patológicas y las propias limitaciones metodológicas de la endoscopia que; no obstante, aún se implementa como enfoque diagnóstico principal para este tipo de condiciones y, en general, en casi todas las formas de HGl.

- Los tratamientos utilizados básicamente consisten en métodos térmicos, de inyección, o mecánicos y ninguno es superior a otro, de hecho, se encontró que es bastante recomendada la terapia combinada de estos para una mayor efectividad. Sin menoscabo, la angiografía terapéutica y la ecografía endoscópica son otras dos alternativas terapéuticas de aceptación.

\section{Bibliografía}

Ansari, P. (2018 de Febrero de 2018). Lesiones vasculares digestivas. Recuperado el 10 de 07 de 2020, de Manual MSD: https://www.msdmanuals. com/es-ve/professional/trastornos-gastrointestinales/hemorragia-digestiva/lesiones-vasculares-digestivas
Baxter, M., \& Aly, E. (2010). Dieulafoy's lesion: current trends in diagnosis and management. Annals. The Royal College Surgeon England, 92(7), 548-554. doi:10.1308/003588410X12699663905311

Botello, Z., Domínguez, L., Núñez, E., González, M., Rosas, V., \& Torices, E. (Octubre-Diciembre de 2013). Tratamiento endoscópico de lesiones de Dieulafoy. Revista de Especialidades Médico-Quirúrgicas, 18(4), 322-327. Recuperado el 11 de 07 de 2020, de http://www.redalyc.org/articulo. oa? $\mathrm{id}=47329250007$

Empendium. (2020). Medicina Interna Basada en la Evidencia 2019/20, On-line. (L. Caro, Editor) Recuperado el 11 de 07 de 2020, de Sitio Web de:: https://empendium.com/manualmibe/chapter/B34. II.4.29.2.

Khan, R., Mahmad, A., Gobrial, M., Onwochei, F., \& Shah, K. (2015). The Diagnostic Dilemma of Dieulafoy's Lesion. Gastroenterol Research, 8(3-4), 201-206. doi:10.14740/gr671w

Ledo Rodríguez, A., \& Ledo Barro, L. (2012). Lesión de Dieulafoy. Revista Española de Enfermedades Digestivas, 104(3), 151-151. doi:10.4321/S113001082012000300010

Massinha, P., Cunha, I., \& Tomé, L. (2020). Dieulafoy Lesion: Predictive Factors of Early Relapse and Long-Term Follow-Up. Portuguese Journal of Gastroenterology, 27(4), 237-243. doi:10.1159/000504720

Rojas, A., Carvajal, G., Prieto, R., \& Aponte, D. (2016). Lesión de Dieulafoy de duodeno: hallazgo inusual. Revista Colombiana de Gastroenterologia, 31(3), 292-296. doi:10.22516/25007440.103

Salazar, E., Galvez, M., Podestá, F., Cinquegrani, K., De María, J., Promenzio, E., \& De Bonis, A. (2017). Lesión de Dieulafoy: reporte de dos casos. Revista del Hospital El Cruce, 21, 45-50. Recuperado el 12 de 07 de 2020, de https://repositorio.hospitalelcruce.org

Soria, M., Betancourt, M., Oleas, R., Baquerizo, J., \& Robles, C. (2018). Clip Ovesco® Como Tratamiento De Rescate En Lesión De Dieulafoy Duodenal Con Sangrado Activo. Revista de Gastroenterol del Peru, 38(4), 374-6. Recuperado el 12 de 07 de 2020, de https://www.netmd.org/gastroenterologia/gastroenterologia-articulos/clip-ovesco-como-tratamiento-de-rescate-en-lesion-de-dieulafoy-duodenal-con-sangrado-activo 


\section{CITAR ESTE ARTICULO:}

Lama Asinc, V., Castro Holguín, M., Morante Mendoza, J., \& García Bermúdes,

A. (2020). Lesión de Dieulafoy. RECIAMUC, 4(3), 4-16. doi:10.26820/reciamuc/4.(3).julio.2020.4-16

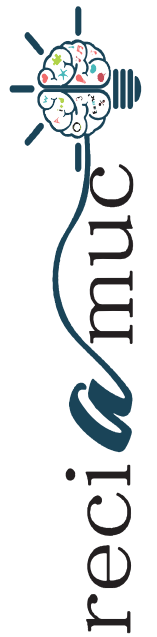

(c) $)$ (1) $\$(0)$

RECONOCIMIENTO-NOCOMERCIAL-COMPARTIRIGUAL CC BY-NC-SA

ESTA LICENCIA PERMITE A OTROS ENTREMEZCLAR, AJUSTAR Y CONSTRUIR A PARTIR DE SU OBRA CON FINES NO COMERCIALES, SIEMPRE Y CUANDO LE RECONOZCAN LA AUTORÍA Y SUS NUEVAS CREACIONES ESTÉN BAJO UNA LICENCIA CON LOS MISMOS TÉRMINOS. 